\title{
Über eine neue Bildungsweise von Thiazolderivaten der Anthrachinonreihe
}

\author{
Von \\ Eduard Kopetschni und Herta Wiesler \\ Aus dem Chemischen Institut der Universität Graz
}

(Vorgeiegt in der Sitzung am 23. Februar 1922)

Im Verlaufe von ausgedehnten Untersuchungen über das 1-Merkapto-2-aminoanthrachinon machten wir die Beobachtung, daß sich dieses bei Einwirkung von alkoholischem oder wässerigem Ammoniak schnell verändert. Zwar bildet sich zunächst das intensiv violettblau gefärbte Ammoniumsalz obiger Merkaptoverbindung, beim Erhitzen dieser Lösung scheidet sich jedoch ein unlösliches gelbes Produkt ab, welches uns durch die auBerordentlich starke Fluoreszenz seiner alkoholischen Lösung auffiel. Wir vermuteten, und dies wurde durch unsere weiteren Untersuchungen bestätigt, in dieser Verbindung das bis nun unbekannte 2-Aminoanthrachinon1-thiazol (II), dessen Bildungsweise aus dem Ammoniumsalz des 1-Merkapto-2-aminoanthrachinons durch nachfolgendes Reaktionsschema veranschaulicht wird:

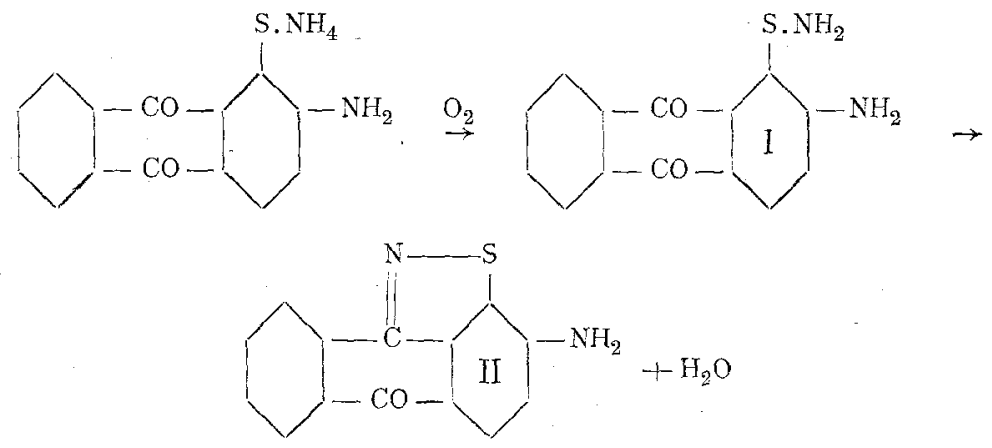


Das bei dieser Reaktion als Zwischenprodukt anzunehmende 2-Amino-1-anthrachinonylschwefelamid (I) ${ }^{1}$ konnten wir $z$ war nicht isolieren, zweifellos aber läuft, wie wir aus einem zu beobachtenden roten Farbenübergang schließen, die Reaktion über Zwischenprodukte.

Der Grundkörper der beschriebenen Verbindung, das Anthrachinon-1-thiazol, wurde zuerst von L. Gattermann ${ }^{2}$ durch Erhitzen von Anthrachinon-1-rhodanid mit wässerigem Ammoniak unter Druck auf $130^{\circ}$ erhalten. Es ist nur schwach gelb gefärbt und zeigt, in organischen Solvenzien gelöst, keine Fluoreszenz. Bei der analogen Umsetzung des 2-Methylanthrachinon-1-rhodanids mit wässerigem Ammoniak konnte Gattermann gleichzeitig auch die Bildung eines Nebenproduktes beobachten, welches sich in Alkohol mit grüner Fluoreszenz löste. Vielleicht steht diese von Gattermann nicht näher untersuchte Substanz mit dem vorstehend beschriebenen 2-Aminoanthrachinon-1-thiazol in einem Zusammenhange, da sich letzteres, wie erwähnt, in Alkohol gleichfalls mit grüner Fluoreszenz löst.

Auch durch direkte Einwirkung von Ammoniak auf 1-Merkaptoanthrachinon unter Druck wurde Anthrachinon-1-thiazol dargestellt, jedoch ist diese Reaktion an die gleichzeitige Anwesenheit von Alkalipolysulfiden ${ }^{3}$ gebunden.

Wie aus dem oben skizzierten Reaktionsschema ersichtlich, ist für die Bildung des 2-Aminoanthrachinon-1-thiazols Sauerstoff erforderlich. Dementsprechend bildet sich beim Kochen von 1*Merkapto-2-aminoanthrachinon mit alkoholisch-wässerigem Ammoniak unter Rückfluß nur zum Teil das Thiazolderivat, während ein Teil der Merkaptoverbindung mit blauer Farbe unverändert in Lösung bleibt. Dampft man jedoch 1-Merkapto-2-aminoanthrachinon bei offenem Luftzutritt. und erhöhter Temperatur (am Sandbade) ab, oder kocht man mit ammoniakalischer Kupfersulfatlösung, so ist die Umsetzung eine vollständige. Dies führte uns zur Vermutung, daßs die Bildung des 2-Aminoanthrachinon-1-thiazols auch aus dem, bei Luftoxydation aus 1-Merkapto-2-aminoanthrachinon zunächst entstehenden 2, $2^{\prime}$-Diaminodianthrachinon-1, $1^{\prime}$-disulfid erfolgen müsse. Diese Annahme wurde durch das Experiment bestätigt. Das erwähnte Disulfid wird beim Erhitzen mit alkoholischem Ammoniak auf $100^{\circ}$ glatt in 2-Aminoanthrachinon-1-thiazol übergeführt. (Siehe nebenstehendes Schema.)

Wir haben in dieser Reaktion einen typischen Kreisprozeß 3 vor uns. Das Disulfid wird durch alkoholisches Ammoniak in 2-Aminoanthrachinon-1-schwefelamid (welches durch Wasserabspaltung in 2-Aminoanthrachinon-1-thiazol übergeht) und 1-Merkapto2 -aminoanthrachinon gespalten. Letzteres wird durch den vorhandenen

\footnotetext{
1 Hierzu vgl. K. Fries und G. Schürmann, Ber., 52 (1919), 2172.

2 Lieb. Ann., 393 (1912), 192.

3 D. R. P. 216306 (Friedl, 9, 743).
} 
Sauerstoff wieder zu Disulfid oxydiert und der geschilderte Prozeß beginnt von neuem bis sämtliches Disulfid in 2-Aminoanthrachinon1 -thiazol übergeführt ist.

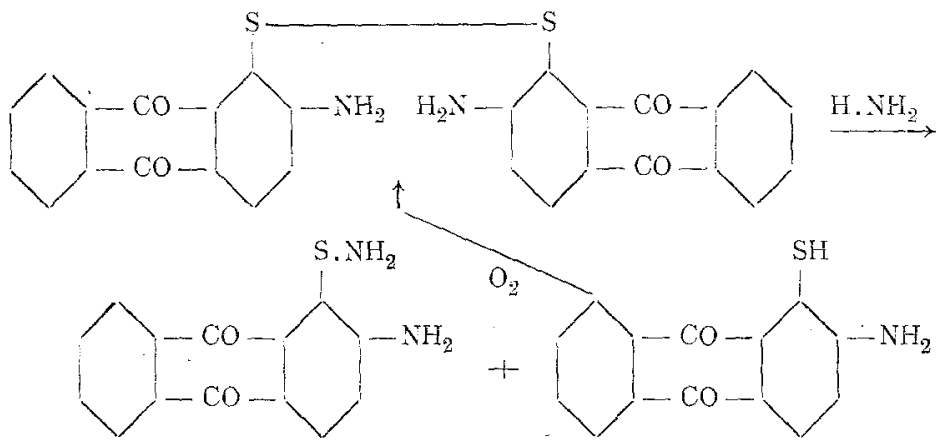

Das 2-Aminoanthrachinon-1-thiazol löst sich in Alkohol mit intensiv grüne: Fluoreszenz die ihrem Charakter und ihrer Stärke nach dem Fluorescein fast gleich kommt. Auf Zusatz von Säure oder Alkali verschwindet die Fluoreszenz. Ebenso zeigt das Acetylderivat keine Fluoreszenz mehr. Von einer salzsauren Suspension des 2-Aminoanthrachinon-1-thiazols wird Wolle oder Seide gelb angefärbt, was als auffallend bezeichnet werden muß. Bemerkenswert ist auch, daB das Thiazolderivat sich mit Hydrosulfit nicht verküpen läßt. Beim Erwärmen mit 20 prozentigem Oleum am Wasserbade erhält man eine Sulfosäure, die Wolle aus sauerem Bade in intensiv gelben, dem Chinolingelb fast gleichen Tönen anfärbt. Die erhaltenen Fürbungen zeigen jedoch nur eine mittlere Lichtechtheit. Wir haben die so erhältliche Sulfosäure nicht näher untersucht, da aber das 3-Chlor-2-aminoanthrachinon1-thiazol, welches wir nach der eben beschriebenen Methode gleichfalls darstellen konnten. beim Erwärmen mit 20 prozentigem Oleum nicht (und mit 70 prozentigem Oieum nur sehr schwer) sulfiert wird, glauben wir behaupten zu können, daß bei der Sulfierung des 2-Aminoanthrachinon-1-thiazols die Sulfogruppe in die 3-Stellung eintritt. Dies würde auch mit dem Verhalten von $\beta$-Aminoanthrachinon, welches bei Sulfierung bekanntlich 2-Aminoanthrachinon-3-sulfosäure gibt, übereinstimmen.

SchlieBlich haben wir unsere Untersuchungen auch auf das unsubstituierte Anthrachinon 1,1'-disulfid ausgedehnt. Ganz analog den beschriebenen Umsetzungen gibt dieses, beim Erhitzen mit alkoholischem Ammoniak, das von Gattermann zuerst dargestellte Anthrachinon 1-thiazol.

Zusammenfassend' ergibt sich daher, daß Anthrachinon 1,1'disulîde beim Erhitzen mit Ammoniak in Anthrachinon 1-thiazole übergehen. Sehr wahrscheinlich erfolgt bei dieser Reaktion eine intermediäre Aufspaltung, was umso bemerkenswerter wäre, als eine Aufspaltung von Disulfiden durch Ammoniak in der Literatur unseres Wissens noch nicht beschrieben ist. 


\section{Experimenteller Teil.}

\section{Darstellung von 1-Merkapto-2-aminoanthrachinon.}

Als Ausgangsmaterial wurde das durch Chlorierung 1 von $\beta$-Acetaminoanthrachinon leicht erhältliche 1-Chlor-2-aminoanthrachinon verwendet. Dieses geht, analog anderen Halogenanthrachinonen $^{2}$ beim Kochen mit polysulfidhältiger Natriumsulfhydratlauge in die entsprechende Merkaptoverbindung über. Da, wie wir konstatieren konnten, für diese Umsetzung einzig der Polysulfidgehalt entscheidend ist, haben wir die Reaktion in der Weise vereinfacht, da $\beta$ wir nur mit Polysulfid arbeiteten. Während bekanntlich ${ }^{3}$ 1-Chloranthrachinon hierbei in normaler Reaktion Dianthrachinon$1,1^{\prime}$-disulfid gibt, erhielten wir aus 1-Chlor-2-aminoanthrachinon glatt das entsprechende Merkaptosalz.

$30 \mathrm{~g}$ 1-Chior-2-aminoanthrachinon wurden mit $400 \mathrm{~cm}^{3}$ Alkohol am Wasserbad rückfließ3end zum Sieden erhitzt und eine Lösung von $10 \mathrm{~g}$ Schwefel in einem Gemisch von $40 \mathrm{~g}$ krystallisiertem Natriumsulfid und $40 \mathrm{~cm}^{3}$ Wasser zugefügt. Nach etwa einstündigem Kochen wurde das in blauvioletten, kupferglänzenden Nadeln abgeschiedene Natriumsalz des 1-Merkapto-2-aminoanthrachinons abgesaugt und mit Alkohol und Äther gewaschen. Die Ausbeute ist quantitativ. Zumeist, namentlich bei größeren Mengen, enthält es jedoch noch etwas unangegriffenes 1-Chlor-2-aminoanthrachinon mechanisch eingeschlossen. In diesem Falle wird das erhaltene Merkaptosalz in einem Gemisch von etwa gleichen Teilen Alkohol und Wasser (eventuell unter Zusatz von etwas Natriumsulfhydratlauge) siedend gelöst, filtriert, das blauviolette Filtrat mit gesättigler. Kochsalzlösung gefällt, abgesaugt und das zurückbleibende Merkaptosalz abermals mit Alkohol und Äther gewaschen. Durch Umsetzung mit verdünnter Salzsäure erhält man daraus das bekannte 1-Merkapto2-aminoanthrachinon, während beim Einleiten von Luft in die heiße alkoholische, alkalisch-wässerige Lösung das entsprechende Disulfid krystallinisch ausgeschieden wird.

\section{Umsetzung von Ammoniak mit 1-Mexkapto-2-aminoanthrachinon.}

$0.5 g$ 1-Merkapto-2-aminoanthrachinon wurden mit alkohopisch wässerigem Ammoniak in einer Porzellanschale am Sandbade wiederholt (bis auf Zusatz von Ammoniak keine Violettfärbung mehr auftrat) zur Trockene gedampft. Es hinterblieb schließlich ein olivgelber Rückstand, der zur Entfernung etwa noch unveränderter

I W. Junghans, Lieb. Ann., 399 (1913), 319.

2 D. R. P. 206536 (Fried1., 9, 703).

3 Ber., 52, (1919), 2176 und Ber., 49 (1918), 793. 
Merkaptoverbindung mit verdünnter Natriumsulfhydratlauge ausgekocht und abgesaugt wurde. Ausbeute: $0.48 \mathrm{~g}$ gleich $97.14 \%$ der Theorie.

Das erhaltene 2-Aminoanthrachinon-1-thiazol wurde aus Aceton in Form mikroskopischer Nädelchen krystallisiert erhalten.

$2.898 \mathrm{mg}$ Substanz gaben $0.299 \mathrm{~cm}^{3} \mathrm{~N}\left(26^{\circ}, 736 \mathrm{~mm}\right)$.

$5.794 \quad \gg \quad 0.593 \mathrm{~cm}^{3} \mathrm{~N}\left(25^{\circ}, 734 \mathrm{~mm}^{\circ}\right)$.

$6.375 \gg \quad$ \$ $.990 \mathrm{mg} \mathrm{BaSO}_{4}$.

$\mathrm{C}_{14} \mathrm{H}_{8} \mathrm{ON}_{2} \mathrm{~S}(252 \cdot 2)$ ber.: $\mathrm{N} 11 \cdot 11, \mathrm{~S} 12 \cdot 72 ;$

gef.: $\mathrm{N} 11 \cdot 40, \mathrm{~N} 11 \cdot 32, \mathrm{~S} 12 \cdot 90$.

In Alkohol und Aceton ist es in der Wärme ziemlich leicht mit gelber Farbe und intensiv grüner Fluoreszenz löslich. In siedendem Toluol ist es nur schwer mit gelber, in Nitrobenzol ziemlich leicht mit olivgelber Farbe, jedoch ohne Fluoreszenz löslich. Konzentrierte Schwefelsäure löst mit intensiv kirschroter Farbe auf Zusatz von Wasser fällt es in gelben Flocken wieder unverändert aus. Der Schmelzpunkt liegt unter Dunkeifärbung und Zersetzung unscharf bei ungefähr $285^{\circ}$.

Mit Essigsäureanhydrid und einem Tropfen Schwefelsäure erhält man daraus das $\beta$-Acetylaminoanthrachinon-1-thiazol. Letzteres wurde aus Nitrobenzol umkrystallysiert und daraus in langen olivgelben, bei $294^{\circ}$ schmelzenden Nadeln erhalten.

$3 \cdot 407 \mathrm{mg}$ Substanz gaben $0.292 \mathrm{~cm}^{3} \mathrm{~N}\left(24^{\circ}, 732 \mathrm{~mm}\right)$.

$\mathrm{C}_{16} \mathrm{H}_{10} \mathrm{O}_{2} \mathrm{~N}_{2} \mathrm{~S}$ (294) ber.: N $9.520 \%$; gef.: $\mathrm{N} 9 \cdot 48$, ber.: C $65 \cdot 26 \%$; gef.: C $65 \cdot 58$, ber.: H $3.43 \%$; gef.: H $3 \cdot 64$.

In siedendem Alkohol oder Toluol ist es nur. sehr schwer mit schwachgelber Farbe ohne Fluoreszenz löslich. Konzentrierte Schwefelsäure löst orangegelb mit grünlicher Fluoreszenz. Durch einstündiges Erwärmen am Wasserbade tritt unter Farbenumschlag von orange in kirschrot Verseifung ein und man erhält beim Verdünnen mit Wasser gelbe Flocken von reinem 2-Aminoanthrachinon1-thiazol. Da letzteres im Gegensatz $z u$ der eben beschriebenen Acetylverbindung nur ungern krystallisiert, haben wir das rohe 2-Aminoanthrachinon-1-thiazol zumeist über die Acetylverbindung gereinigt.

\section{Umsetzung von 2, 2'-Diaminodianthrachinon-1, 1'-disulfid mit Ammoniak.}

$3 \cdot 8$ g $2,2^{\prime}$-Diaminodianthrachinon-1, $1^{\prime}$-disulfid wurden mit alkoholischem Ammoniak im Bombenrohr 3 Stunden $100^{\circ}$ erhitzt. Das in Form gelber Nadeln erhaltene Reaktionsprodukt $(2 \cdot 9 \mathrm{~g})$ 
abgesaugt, nach dem Trocknen mit Essigsäureanhydrid acetyliert, mit Wasser verdünnt und abgesaugt. Es hinterblieben $3.1 \mathrm{~g}$ der in Form hellgelber Nadeln krystallisierten Acetylverbindung, aus der durch Verseifung $2 \cdot 6 g(68 \cdot 97 \%$ der Theorie) reines 2-Aminoanthrachinon-1-thiazol gewonnen wurden. Ein zweiter Versuch ergab eine Ausbeute von $81 \%$ der Theorie.

\section{Umsetzung von 3, 3'-Dichlor-2, 2'-diaminodianthrachinon- 1, $1^{\prime}$-disulfid mit Ammoniak.}

Analog der früher beschriebenen Umsetzung läßt sich aus dem bekannten 1,3-Dichlor-2-aminoanthrachinon das entsprechende Disulfid gewinnen, jedoch genügt hier zur Überführung der Merkaptoverbindung in das Disulfid nicht mehr der Luftsauerstoff, sondern man muß mit Hilfe von Oxydationsmitteln (Ammonpersulfat, Ferricyankalium) arbeiten.

$2 \cdot 3 \mathrm{~g} 3,3^{\prime}$-Dichlor-2, 2'-diaminodianthrachinon-1, 1'-disulfid wurden mit alkoholischem Ammoniak (auf dessen Zusatz; wahrscheinlich infolge sofort eintretender Aufspaltung, vorübergehend eine violettblaue Lösungsfarbe auftritt) im zugeschmolzenen Rohr 3 Stunden auf $100^{\circ}$ erhitzt. Nach dem Erkalten bestand der Röhreninhalt aus einem Aggregat von langen, glänzenden, bläulichgrün schimmernden Nadeln. Dieselben wurden abgesaugt und mit Alkohol gewaschen.

Ausbeute: $1.6 \mathrm{~g}$ gleich $69.9 \%$ der Theorie.

Aus Nitrobenzol krystallisiert die Verbindung in großen bläulichmetallisch glänzenden (unter dem Mikroskop olivgrün gefärbten) Prismen, die bei 322 bis $324^{\circ}$ unter Dunkelfärbung schmelzen. $3.690 \mathrm{~g}$ Substanz gaben $0.318 \mathrm{~cm}^{3} \mathrm{~N}\left(23^{\circ}, 736 \mathrm{~mm}\right)$.

$\mathrm{C}_{14} \mathrm{H}_{7} \mathrm{ON}_{2} \mathrm{ClS}(286 \cdot 5)$ ber.: N $9 \cdot 77$; gef.: N $9 \cdot 62$.

In Alkohol ist die Verbindung sehr schwer mit gelber Farbe und grüner Fluoreszenz löslich. Siedendes Xylol löst schwer, Nitrobenzol ziemlich leicht mit olivgrüner Farbe. Konzentrierte Schwefelsäure löst mit intensiv fuchsinroter Farbe und auf Zusatz von Wasser fällt die unveränderte Verbindung in bräunlichen Flocken, welche beim Stehen oder kurzem Erwärmen hellgrünlichgelb (krystallinisch?) werden, wieder aus.

Im Gegensatz zum 2-Aminoanthrachinon-1-thiazol wird es beim Erwärmen mit 20 prozentigem Oleum am Wasserbade nicht sulfiert.

\section{Umsetzung von Dianthrachinon 1,1'-disulfid mit Ammoniak.}

$4 g$ Dianthrachinon 1,1'-disulfid wurden mit alkoholischem Ammoniak im Bombenrohr' (halb gefüllt) 4 Stunden auf $100^{\circ}$ erhitzt. Das erhaltene hellgelbe krystallinische Reaktionsprodukt wurde 
von der dunklen, schwach grünlich fluoreszierenden Mutterlauge abgesaugt und zuerst mit heißer alkalisch-wässriger Hydrosulfilösung, dann mit Alkohol gewaschen.

Ausbeute: $3 \cdot 4 g$ gleich $88 \%$ der Theorie.

Durch Krystallisation aus Nitrobenzol oder Eisessig erhält man reines Anthrachinon 1-thiazol in Form hellgelber, mikroskopischer Prismen, welche in Übereinstimmung mit den Angaben Gattermann's, bei $221^{\circ}$ schmelzen. Das Anthrachinon 1-thiazol läßt sich, gleich den früher beschriebenen Derivaten, mit Hydrosulfit nicht verküpen. Konzentrierte Schwefelsäure löst mit intensiv gelber Farbe und schwach grünlicher Fluoreszenz.

Dem hochverehrten Vorstand des hiesigen Chemischen Institutes, Herrn Prof. Dr. Anton Skrabal, bin ich für die Überlassung eines Arbeitsplatzes sowie das stets bewiesene weitgehende Entgegenkommen zu größtem Danke verpflichtet. Ferner ist es mir eine angenehme Pflicht, den Höchster Farbwerken für die freundliche Überlassung von Ausgangsmaterial für vorliegende Untersuchungen auch an dieser Stelle bestens zu danken.

Eduard Kopetschni. 